\title{
Activation Energy of Modified Peak Shape Equations
}

\author{
Hugo A. Borbón-Nuñez1*, Claudio Furetta² \\ ${ }^{1}$ CONACYT-Centro de Nanociencias y Nanotecnología, UNAM, Ensenada B.C., Mexico \\ ${ }^{2}$ Retired from Physics Department of Rome University, Rome, Italy \\ Email: ^hborbon@cnyn.unam.mx
}

How to cite this paper: Borbón-Nuñez, H.A. and Furetta, C. (2017) Activation Energy of Modified Peak Shape Equations. World Journal of Nuclear Science and Technology, 7, 274-283.

https://doi.org/10.4236/wjinst.2017.74021

Received: July 4, 2017

Accepted: September 17, 2017

Published: September 20, 2017

Copyright (c) 2017 by authors and Scientific Research Publishing Inc. This work is licensed under the Creative Commons Attribution International License (CC BY 4.0).

http://creativecommons.org/licenses/by/4.0/

c) (i) Open Access

\begin{abstract}
The aim of this paper is to give some simplified expressions related to the peak shape method. The modified equations have been used to calculate the activation energy $(E)$ of commercial thermoluminescent dosimeters (TLD), as well as of $\mathrm{ZnO}$ thermoluminescent material produced in laboratory; the values so determined have been compared to the values obtained using the classical expressions of the peak shape method. The modified equations proposed are as a function of peak shape parameters or the peak temperature at the maximum. This expression could be useful to obtain approximated $\mathrm{E}$ values in the case of complex glow curves as well, when the peaks are not well resolved but the peak temperature at the maximum may be easily determined.
\end{abstract}

\section{Keywords}

Thermoluminescence, Geometrical Parameters, Activation Energy, Peak-Shape Method

\section{Introduction}

Luminescence is defined as the emission of light from some solids commonly called phosphors. This emission is the release of energy stored within the solid through some type of prior excitation of the solid electronic system, i.e. by visible, infrared (IR) or ultra violet (UV) light and ionizing radiation [1] [2]. The wavelength of the emitted light is a characteristic of the luminescent material [2]. The ability to store the radiation energy is important in luminescence dosimetry and is generally associated with the presence of activators (i.e. impurity atoms and structural defects) which act as trapping levels for the free electrons generated by excitation [1] [3].

The luminescence effect can be used in solid state dosimetry for measurement 
of ionizing radiation dose; the most popular luminescence dosimetric technique is called thermoluminescence (TL): it consists in a transient emission of light from an irradiated solid when heated [1] [3].

Thermoluminescence is characterized by the following main points:

1) Transient effects, superimposed on an intrinsic background;

2) A memory but not a permanent record of the irradiation dose.

Materials of interest in thermoluminescent dosimetry (TLD) are principally insulators in which the conduction electrons are entirely due to the absorbed radiation energy.

Thermoluminescence is observed under condition of steadily increasing temperature. In the usual thermoluminescence experiments, the TL material is irradiated at room temperature (RT) and later heated through a temperature range where the luminescence is bright, until a temperature level at which all the charges have been thermally excited out of their metastable levels and the luminescence completely disappears [4].

If the light intensity is plotted as a function of temperature (or time), the resulting graph is called glow-curve (Figure 1). The glow-curves have one or more maxima called glow-peaks which correspond to the various energy level traps [1] [3].

In the field of TL dosimetry, the analysis of multipeak glow curves includes the evaluation of the kinetics parameters $E$ (activation energy) and $s$ (frequency factor) related to each TL peak. Several methods to determine these kinetics parameters, mainly the activation energy, have been proposed, based on one-trap models (related to a single peak), and hence the applicability of these methods to multi-peak glow curves is limited for the complexity of the glow curves shape [5] [6] [7] [8]. Among the most applied methods to evaluate the activation energy or trap depth from experimental glow curves are: initial rise method [5], variable heating rate methods [9] and peak shape methods [10]. Also the use of computerized glow curve deconvolution is a widely used method to evaluate the kinetic

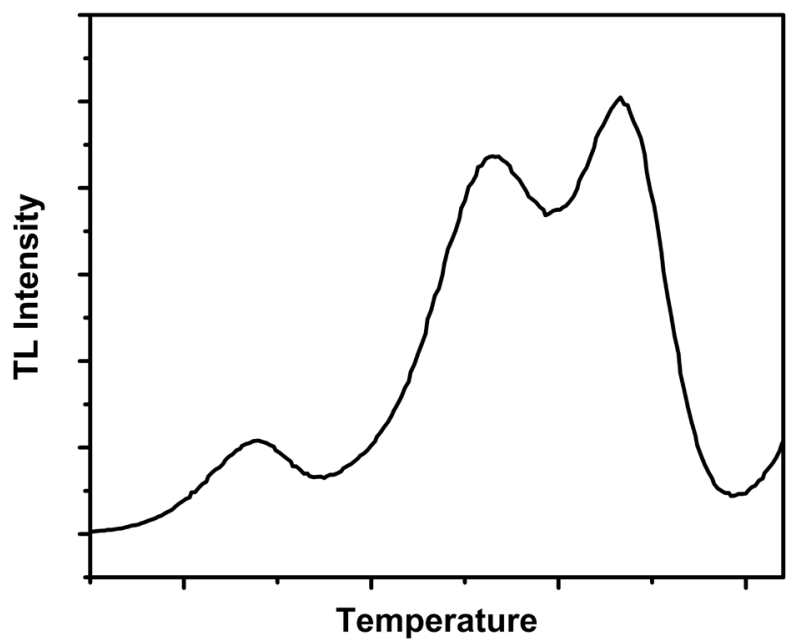

Figure 1. Glow curve example. 
parameters of a glow curve [11]. The peak shape methods are the most employed due to their simplicity and accuracy on activation energies values obtained. However, when a complex glow curve is presented, it is not easy to evaluate accurately the geometrical parameters on individual peaks, and therefore, calculate the activation energies for the related traps.

The aim of this paper is to propose some simplified expressions related to the peak shape method. The modified equations have been used to calculate the activation energy, when the peaks are not well resolved but the peak temperature at the maximum may be easily determined.

\section{Thermoluminescence Equations}

This paper presents simulated glow peaks obtained using the computation of the first order kinetics equation, i.e. the Randall-Wilkins equation [5]

$$
I(T)=n_{0} s \exp \left(-\frac{E}{k T}\right) \exp \left[-\frac{s}{\beta} \int_{T_{0}}^{T} \exp \left(-\frac{E}{k T^{\prime}}\right) \mathrm{d} T^{\prime}\right]
$$

where, $n_{0}$ is the initial concentration of filled traps; $s$ is a pre-exponential frequency factor; $E$ is the activation energy or trap depth; $k$ is Boltzmann's constant; $T$ is the absolute temperature and $\beta$ is the heating rate.

For the second order equation, i.e. the Garlick-Gibson equation [6]

$$
I(T)=\frac{n_{0} s \exp \left(-\frac{E}{k T}\right)}{\left[1+\frac{s}{\beta} \int_{T_{0}}^{T} \exp \left(-\frac{E}{k T^{\prime}}\right) \mathrm{d} T^{\prime}\right]^{2}}
$$

and, finally, the general order equation, i.e. the May-Partridge equation [7]

$$
I(T)=s n_{0} \exp \left(-\frac{E}{k T}\right)\left[1+\frac{s(b-1)}{\beta} \int_{T_{0}}^{T} \exp \left(-\frac{E}{k T^{\prime}}\right) \mathrm{d} T^{\prime}\right]^{-\frac{b}{b-1}}
$$

where, $b$ the kinetic order, a parameter with values typically between 1 and 2 .

The shape of the glow peak is different according to the kinetic process, as is observed in Figure 2, for a simulated glow curve, where a first-order peak has an asymmetrical shape, while a second-order peak is characterized by a symmetrical shape.

An isolated TL glow peak, obtained using a linear heating rate, without the kinetic order it follows, can be characterized by some geometrical parameters $T_{M}, T_{1}, T_{2}, \tau, \delta$ and $\theta$, as it can be seen in Figure 3. $T_{M}, T_{1}, T_{2}$ : are respectively the peak temperature at the maximum and the temperatures on either side of the temperature at the maximum, corresponding to half intensity. The geometrical parameters from a peak are evaluated as follows:

- $\tau=T_{M}-T_{1}$ : is the half-width at the low temperature side of the peak,

- $\delta=T_{2}-T_{M}$ : is the half-width towards the fall-off of the glow peak,

- $\omega=T_{2}-T_{1}$ : is the total half-width,

- $\mu_{g}=\delta / \omega$ : is the so called symmetrical geometrical factor. 


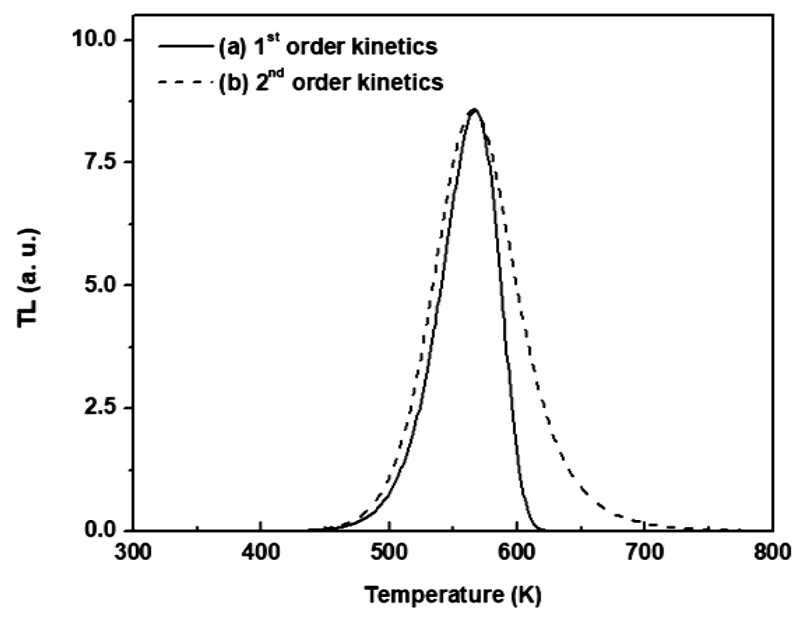

Figure 2. Variation of glow peak shape according to the kinetic process.

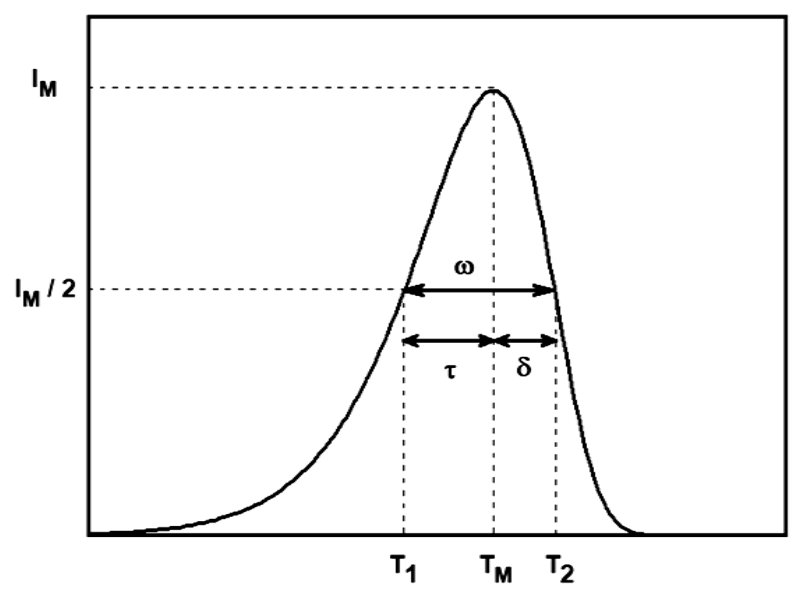

Figure 3. The geometrical parameters characterizing an isolated peak.

Considering the so given characteristic temperatures of a glow peak, glow peaks of both first and second order kinetics have been generated by computer using Equations (1) and (2) and the following relations among the peak temperatures have been obtained:

- In case of first and second order peaks: $T_{1}=0.95029 T_{M}$

- For a first order peak: $T_{2}=1.03132 T_{M}$

- For a second order peak: $T_{2}=1.05648 T_{M}$

It has also to be noted that:

- According to the asymmetrical property on a glow curve shape, for a first-order peak $\tau$ is almost $40 \%$ bigger than $\delta$, due the re-trapping process involved.

- The geometrical factor $\mu_{g}$ is equal to 0.42 for first-order kinetics, and 0.52 in the case of second-order.

- $\mu_{g}$ is practically independent of $E$, in the range from 0.1 to $1.6 \mathrm{eV}$.

- $\mu_{g}$ is strongly dependent on the kinetics order, $b$, in the range $0.7 \leq b \leq 2.5$,

- Another factor, namely $\gamma=\delta / \tau$, is ranging from 0.7 to 0.8 for a first-order 
peak, and from 1.05 to 1.20 for a second-order.

An important and widely used method to investigate the trapping levels in crystals is based on the geometrical characterization of a TL glow peak, the well-known peak shape methods. In fact, for calculating the activation energy of the trapping level corresponding to a peak in the glow curve, one needs to measure three temperature values on the peak itself: $T_{M}, T_{I}$ and $T_{2}$. The equations for determining the activation energy according to the peak shape methods are the followings:

Grossweiner was the first to use the shape of the glow curve to calculate the trap depth and frequency factor [12]. His method is based on the temperature at the maximum and on the low temperature at half intensity, $T_{l}$. The expressions to evaluate $E$, as revised by Chen [13], are:

$$
\begin{aligned}
1^{\text {st }} \text { order }\left(E_{G}\right)_{\tau} & =1.41 k \frac{T_{1} T_{M}}{\tau} \\
2^{\text {nd }} \text { order }\left(E_{G}\right)_{\tau} & =1.68 k \frac{T_{1} T_{M}}{\tau}
\end{aligned}
$$

Lushchik also proposed a method based on the shape for first- and second-order kinetics introducing the parameter $\delta$ [14]. The expressions are the follows:

$$
\begin{aligned}
1^{\text {st }} \text { order }\left(E_{L}\right)_{\delta} & =0.976 \frac{k T_{M}^{2}}{\delta} \\
2^{\text {nd }} \operatorname{order}\left(E_{L}\right)_{\delta} & =1.706 \frac{k T_{M}^{2}}{\delta}
\end{aligned}
$$

where the coefficients have been computed by Chen [13].

Halperin \& Braner gave a different approach by using both $T_{1}$ and $T_{2}$. They consider the luminescence emission as mainly due to different kinds of recombination processes [15]. In the first one the electrons raise to an excited state within the band gap below the conduction band and recombine with holes by the tunneling process. In the second process, the recombination takes place via the conduction band. The equations proposed by Halperin \& Braner introducing the parameter $\tau$, for the two different kinetics are

$$
\begin{gathered}
1^{\text {st }} \text { order }\left(E_{H B}\right)_{\tau}=1.72 \frac{k T_{M}^{2}}{\tau}\left(1-1.58 \Delta_{M}\right) \\
2^{\text {nd }} \text { order }\left(E_{H B}\right)_{\tau}=\frac{2 k T_{M}^{2}}{\tau}\left(1-3 \Delta_{M}\right)
\end{gathered}
$$

where $\Delta_{M}=\frac{2 k T_{M}}{E}$.

Both the previous Equations (9) and (10) require an iterative process owing the presence of the quantity $\Delta_{\mathrm{M}}$. To avoid this difficulty, Chen modified them as following [13]:

$$
1^{\text {st }} \text { order }\left(E_{H B}\right)_{\tau}=1.52 \frac{k T_{M}^{2}}{\tau}-3.16 k T_{M}
$$




$$
2^{\text {nd }} \text { order }\left(E_{H B}\right)_{\tau}=1.813 \frac{k T_{M}^{2}}{\tau}-4 k T_{M}
$$

Chen also gave two expressions, using numerical approximations and without knowledge of the kinetics order which is directly found from the peak shape. Based on the Lushchik, Halperin and Braner previous studies, he proposed the following equations based on parameter $\omega$ [13]:

$$
\begin{aligned}
& 1^{s t} \text { order }\left(E_{H B}\right)_{\omega}=2 k T_{M}\left(1.25 \frac{T_{M}}{\omega}-1\right) \\
& 2^{\text {nd }} \text { order }\left(E_{H B}\right)_{\omega}=2 k T_{M}\left(1.76 \frac{T_{M}}{\omega}-1\right)
\end{aligned}
$$

$\underline{\text { Balarin }}$ also gave expressions based on the quantity $\omega$, [16] [17], values with an accurately to within $0.5 \%$.The expressions are:

$$
\begin{aligned}
1^{\text {st }} \text { order }\left(E_{B}\right)_{\omega} & =\frac{T_{M}^{2}}{\omega \cdot 4998} \\
2^{\text {nd }} \operatorname{order}\left(E_{B}\right)_{\omega} & =\frac{T_{M}^{2}}{\omega \cdot 3542}
\end{aligned}
$$

\section{Modified Equations}

Considering the three temperature values characterizing a TL peak, i.e. $T_{M}, T_{1}$ and $T_{2}$ and the asymmetrical and symmetrical characteristics of the glow peaks following first or second order kinetics respectively, synthetic peaks have been generated, giving the temperature at the maximum, $T_{M}$, ranging from $300 \mathrm{~K}$ to $750 \mathrm{~K}$.

In this way, the original equations used in the peak shape method, from Equation (6) to Equation (17), have been modified using a little algebra and the following new equations have been obtained as a function of the peak temperatures already defined trough Equations (4) and (5).

The modified equations were evaluated as a function of the peak temperature parameters and of the peak temperature at the maximum, as follow:

\begin{tabular}{llll}
\hline & Kinetic order & Equation & \\
\hline Grosswiener & $1^{\text {st }}$ kinetic order & $\mathrm{E}=0.0462 \tau$ & $(18 \mathrm{a})$ \\
& $2^{\text {nd }}$ kinetic order & $\mathrm{E}=0.055 \tau$ & $(18 \mathrm{~b})$ \\
Lushchik & $1^{\text {st }}$ kinetic order & $\mathrm{E}=0.0649 \delta$ & $(19 \mathrm{a})$ \\
& $2^{\text {nd }}$ kinetic order & $\mathrm{E}=0.0504 \delta$ & $(19 \mathrm{~b})$ \\
Halperin and Braner & $1^{\text {st }}$ kinetic order & $\mathrm{E}=0.0732 \tau$ & $(20 \mathrm{a})$ \\
& $2^{\text {nd }}$ kinetic order & $\mathrm{E}=0.0460 \tau$ & $(20 \mathrm{~b})$ \\
Balarin & $1^{\text {st }}$ kinetic order & $\mathrm{E}=0.0271 \omega$ & $(21 \mathrm{a})$ \\
& $2^{\text {nd }}$ kinetic order & $\mathrm{E}=0.0261 \omega$ & $(21 \mathrm{~b})$ \\
\hline
\end{tabular}

Also, the modified equations were evaluated as a function of the peak temperature at the maximum, as follow: 


\begin{tabular}{llll}
\hline & Kinetic Order & Equation & \\
\hline Grosswiener & $1^{\text {st }}$ kinetic order & $\mathrm{E}=2.323 \times 10^{-3} \mathrm{~T}_{\mathrm{M}}$ & $(18 \mathrm{c})$ \\
& $2^{\text {nd }}$ kinetic order & $\mathrm{E}=2.768 \times 10^{-3} \mathrm{~T}_{\mathrm{M}}$ & $(18 \mathrm{~d})$ \\
Lushchik & $1^{\text {st }}$ kinetic order & $\mathrm{E}=2.685 \times 10^{-3} \mathrm{~T}_{\mathrm{M}}$ & $(19 \mathrm{c})$ \\
& $2^{\text {nd }}$ kinetic order & $\mathrm{E}=2.603 \times 10^{-3} \mathrm{~T}_{\mathrm{M}}$ & $(19 \mathrm{~d})$ \\
Halperin and Braner & $1^{\text {st }}$ kinetic order & $\mathrm{E}=2.363 \times 10^{-3} \mathrm{~T}_{\mathrm{M}}$ & $(20 \mathrm{c})$ \\
& $2^{\text {nd }}$ kinetic order & $\mathrm{E}=2.788 \times 10^{-3} \mathrm{~T}_{\mathrm{M}}$ & $(20 \mathrm{~d})$ \\
Balarin & $1^{\text {st }}$ kinetic order & $\mathrm{E}=2.574 \times 10^{-3} \mathrm{~T}_{\mathrm{M}}$ & $(21 \mathrm{c})$ \\
& $2^{\text {nd }}$ kinetic order & $\mathrm{E}=2.579 \times 10^{-3} \mathrm{~T}_{\mathrm{M}}$ & $(21 \mathrm{~d})$ \\
\hline
\end{tabular}

\section{Discussion}

The following Table 1 reports some data concerning the range of the activation energy for two different materials, $\mathrm{CaF}_{2}: \mathrm{Tm}$ (TLD-300) [14] and $\mathrm{BeO}$ [15], obtained using several methods including the initial rise, the isothermal decay and the fitting method. In the same table are also reported the averaged $E$ values obtained using the simplified expressions presented in this work.

With the aim to apply the modified equations on a glow curve having peaks not well resolved, the values of $E$ of a $\mathrm{ZnO}$ thermoluminescent material have been calculated, and then compared to the values obtained using the original peak shape expression, reported on a previous work [18].

In Table 2 are presented the activation energy values calculated for each individual $\mathrm{TL}$ peak of $\mathrm{ZnO}$ samples by peak shapes methods, from the $\mathrm{ZnO}$

Table 1. Activation energies of $\mathrm{CaF}_{2}: \mathrm{Tm}$ and $\mathrm{BeO}$.

\begin{tabular}{cccccc}
\hline Phosphor & Reference & $\begin{array}{c}E(\mathrm{eV}) \\
\text { 1st peak }\end{array}$ & $\begin{array}{c}E(\mathrm{eV}) \\
\text { 2nd peak }\end{array}$ & $\begin{array}{c}E(\mathrm{eV}) \\
\text { 3rd peak }\end{array}$ & $\begin{array}{c}E(\mathrm{eV}) \\
\text { 5th peak }\end{array}$ \\
\hline CaF2:Tm (TLD-300) & {$[14]$} & $0.69 \div 0.74$ & & $1.07 \div 1.31$ & $1.68 \div 1.90$ \\
& This work & $0.91 \div 1.04$ & & $0.91 \div 1.18$ & $1.35 \div 1.45$ \\
$\mathrm{BeO}$ & {$[15]$} & $0.99 \div 1.55$ & $1.16 \div 1.35$ & & \\
& This work & $1.17 \div 1.38$ & $1.49 \div 1.72$ & & \\
\hline
\end{tabular}

Table 2. Activation energy $E$ calculated for each individual TL peak of $\mathrm{ZnO}$ samples by peak shapes methods.

\begin{tabular}{|c|c|c|c|c|}
\hline Method & Kinetic Order & $\begin{array}{c}\text { Activation energy, E } \\
(\mathrm{eV}) \text { 1st Peak }\end{array}$ & $\begin{array}{c}\text { Activation energy, } \\
\text { E (eV) 2nd Peak }\end{array}$ & $\begin{array}{c}\text { Activation energy, } \\
\text { E (eV) 3rd Peak }\end{array}$ \\
\hline \multirow{2}{*}{ Grossweiner } & 1st Order & 0.54 & 0.74 & 0.99 \\
\hline & 2nd Order & 0.65 & 0.85 & 1.18 \\
\hline \multirow{2}{*}{ Lushchik } & 1st Order & -- & -- & 1.07 \\
\hline & 2nd Order & -- & -- & 1.88 \\
\hline \multirow{2}{*}{$\begin{array}{l}\text { Halperin- } \\
\text { Braner }\end{array}$} & 1st Order & 0.55 & 0.77 & 1.03 \\
\hline & 2nd Order & 0.62 & 0.88 & 1.17 \\
\hline \multirow{2}{*}{ Balarin } & 1st Order & -- & -- & 0.96 \\
\hline & 2nd Order & -- & -- & 1.04 \\
\hline
\end{tabular}


experimental glow curve (Figure S1). Table 3 and Table 4, show the activation energy values calculated with the modified peak shape equations for both first and second order kinetics, as a function of the peak temperature parameters, as well as a function of the peak temperature at the maximum. The corresponding standard deviation has been also calculated.

As it can be observed from the Tables, the $E$ values calculated using the classical methods, i.e. Equations (6) to (17) are not much different from the ones obtained using the modified equations here proposed.

For the expressions as a function of the peak temperature parameters, the $E$ values for low temperature peaks are higher than the values obtained from the

Table 3. Activation energies of $\mathrm{ZnO}$ from modified peak shape equations for both first and second order kinetics, as a function of the peak temperature parameters $\tau, \delta$ and $\omega$, and standard deviation from values obtained by peak shape formulas.

\begin{tabular}{|c|c|c|c|c|c|c|c|}
\hline Method & $\begin{array}{c}\text { Kinetic } \\
\text { Order }\end{array}$ & $\begin{array}{c}\text { Activation } \\
\text { energy, E }(\mathrm{eV}) \\
\text { 1st Peak }\end{array}$ & $\begin{array}{l}\text { Standard } \\
\text { Deviation }\end{array}$ & $\begin{array}{c}\text { Activation } \\
\text { energy, E } \\
\qquad(\mathrm{eV}) \\
\text { 2nd Peak }\end{array}$ & $\begin{array}{l}\text { Standard } \\
\text { Deviation }\end{array}$ & $\begin{array}{c}\text { Activation } \\
\text { energy, E } \\
(\mathrm{eV}) \\
\text { 3rd Peak }\end{array}$ & $\begin{array}{l}\text { Standard } \\
\text { Deviation }\end{array}$ \\
\hline \multirow{2}{*}{ Grossweiner } & 1st Order & 1.48 & 0.66 & 1.84 & 0.77 & 1.75 & 0.53 \\
\hline & 2nd Order & 1.76 & 0.78 & 2.2 & 0.95 & 2.09 & 0.64 \\
\hline \multirow{2}{*}{ Lushchik } & 1st Order & -- & -- & -- & -- & 1.68 & 0.43 \\
\hline & 2nd Order & -- & -- & -- & -- & 1.31 & 0.40 \\
\hline \multirow{2}{*}{$\begin{array}{l}\text { Halpe- } \\
\text { rin-Braner }\end{array}$} & 1st Order & 2.34 & 1.26 & 2.92 & 1.52 & 2.78 & 1.23 \\
\hline & 2nd Order & 1.47 & 0.60 & 1.84 & 0.67 & 1.74 & 0.40 \\
\hline \multirow{2}{*}{ Balarin } & 1st Order & -- & -- & -- & -- & 1.73 & 0.54 \\
\hline & 2nd Order & -- & -- & -- & -- & 1.67 & 0.44 \\
\hline
\end{tabular}

Table 4. Activation energies of $\mathrm{ZnO}$ from modified peak shape equations for both first and second order kinetics, as a function of the peak temperature at the maximum, and standard deviation from values obtained by peak shape formulas.

\begin{tabular}{|c|c|c|c|c|c|c|c|}
\hline Method & $\begin{array}{c}\text { Kinetic } \\
\text { Order }\end{array}$ & $\begin{array}{c}\text { Activation } \\
\text { energy, E } \\
(\mathrm{eV}) \\
\text { 1st Peak }\end{array}$ & $\begin{array}{l}\text { Standard } \\
\text { Deviation }\end{array}$ & $\begin{array}{c}\text { Activation } \\
\text { energy, E (eV) } \\
\text { 2nd Peak }\end{array}$ & $\begin{array}{c}\text { Standard } \\
\text { Devia- } \\
\text { tion }\end{array}$ & $\begin{array}{c}\text { Activation } \\
\text { energy, E (eV) } \\
\text { 3rd Peak }\end{array}$ & $\begin{array}{l}\text { Standard } \\
\text { Deviation }\end{array}$ \\
\hline \multirow{2}{*}{ Grossweiner } & 1st Order & 0.92 & 0.26 & 1.19 & 0.31 & 1.34 & 0.24 \\
\hline & 2nd Order & 1.09 & 0.31 & 1.42 & 0.40 & 1.60 & 0.29 \\
\hline \multirow{2}{*}{ Lushchik } & 1st Order & 1.06 & -- & 1.38 & -- & 1.55 & 0.33 \\
\hline & 2nd Order & 1.03 & -- & 1.34 & -- & 1.50 & 0.26 \\
\hline \multirow{2}{*}{$\begin{array}{l}\text { Halperin- } \\
\text { Braner }\end{array}$} & 1st Order & 0.93 & 0.26 & 1.21 & 0.31 & 1.36 & 0.23 \\
\hline & 2nd Order & 1.10 & 0.33 & 1.43 & 0.38 & 1.61 & 0.31 \\
\hline \multirow{2}{*}{ Balarin } & 1st Order & 1.02 & -- & 1.32 & -- & 1.48 & 0.36 \\
\hline & 2nd Order & 1.02 & -- & 1.33 & -- & 1.49 & 0.31 \\
\hline
\end{tabular}


original equations. As the $T_{M}$ value increases the standard deviation decreases and, furthermore, the $E$ values are more closed to the values obtained before.

\section{Conclusions}

In any case, the expressions proposed could be useful to obtain approximated $E$ values in the case of complex glow curves, when the peaks are not well resolved but the peak temperature at the maximum may be easily determined; furthermore, the approximated $E$ values so determined may be inserted in a deconvolution programmer which allows a more precise determination of the kinetics parameters.

\section{References}

[1] Furetta, C. and Weng, P.-S. (1998) Operational Thermoluminescence Dosimetry. World Scientific, Singapore, 260. https://doi.org/10.1142/3789

[2] Kitai, A. (2008) Luminescent Materials and Applications. John Wiley \& Sons, Ltd., New York. https://doi.org/10.1002/9780470985687

[3] McKeever, S.W.S. (2011) Thermoluminescence Dosimetry (TLD). In: Thermolumin Solids, Cambridge University Press, Cambridge, 205-252.

https://doi.org/10.1017/CBO9780511564994

[4] Azorín, J., Furetta, C. and Scacco, A. (1993) Preparation and Properties of Thermoluminescent Materials. Physica Status Solidi $(A), 138,9-46$.

https://doi.org/10.1002/pssa.2211380102

[5] Randall, J.T. and Wilkins, M.H.F. (1945) The Phosphorescence of Various Solids. Proceedings of the Royal Society of London, Series A, Mathematical and Physical Sciences, 184, 347LP-364LP. https://doi.org/10.1098/rspa.1945.0023

[6] Garlick, G.F.J. and Gibson, A.F. (1948) The Electron Trap Mechanism of Luminescence in Sulphide and Silicate Phosphors. Proceedings of the Physical Society, 60, 574-590. https://doi.org/10.1088/0959-5309/60/6/308

[7] May, C.E. and Partridge, J.A. (1964) Thermoluminescent Kinetics of Alpha-Irradiated Alkali Halides. The Journal of Chemical Physics, 40, 1401-1409. https://doi.org/10.1063/1.1725324

[8] McKeever, S.W.S., Moscovitch, M. and Townsend, P.D. (1995) Thermoluminescence Dosimetry Materials: Properties and Uses. Journal of Radiological Protection, 16, No. 4.

[9] Chen, R. and Winer, S.A.A. (1970) Effects of Various Heating Rates on Glow Curves. Journal of Applied Physics, 41, 5227-5232. https://doi.org/10.1063/1.1658652

[10] Furetta, C. (2003) Handbook of Thermoluminescence. World Scientific, Singapore. https://doi.org/10.1142/5167

[11] Gómez Ros, J.M. and Kitis, G. (2002) Computerised Glow Curve Deconvolution using General and Mixed Order Kinetics. Radiation Protection Dosimetry, 101, 47-52. https://doi.org/10.1093/oxfordjournals.rpd.a006029

[12] Grossweiner, L.I. (1953) A Note on the Analysis of First-Order Glow Curves. Journal of Applied Physics, 24, 1306. https://doi.org/10.1063/1.1721152

[13] Chen, R. (1969) On the Calculation of Activation Energies and Frequency Factors from Glow Curves. Journal of Applied Physics, 40, 570-585.

https://doi.org/10.1063/1.1657437 
[14] Lushchik, C.B. (1956) The Investigation of Trapping Centers in Crystals by the Method of Thermal Bleaching. Soviet Physics-JETP, 3, 390-399.

[15] Halperin, A. and Braner, A.A. (1960) Evaluation of Thermal Activation Energies from Glow Curves. Physical Review, 117, 408-415.

https://doi.org/10.1103/PhysRev.117.408

[16] Balarin, M. (1975) Direct Evaluation of Activation Energy from Half-Width of Glow Peaks and a Special Nomogram. Physica Status Solidi, 31, K111-K114. https://doi.org/10.1002/pssa.2210310259

[17] Balarin, M. (1979) Half-Width and Asymmetry of Glow Peaks and Their Consistent Analytical Representation. Journal of Thermal Analysis and Calorimetry, 17, 319-332. https://doi.org/10.1007/BF01914023

[18] Borbón-Nuñez, H.A., Iriqui-Razcón, J.L., Cruz-Vázquez, C., Bernal, R., Furetta, C., Chernov, V. and Castaño, V.M. (2017) Thermoluminescence Kinetics Parameters of $\mathrm{ZnO}$ Exposed to Beta Particle Irradiation. Journal of Materials Science, 52, 5208-5215. https://doi.org/10.1007/s10853-017-0761-y

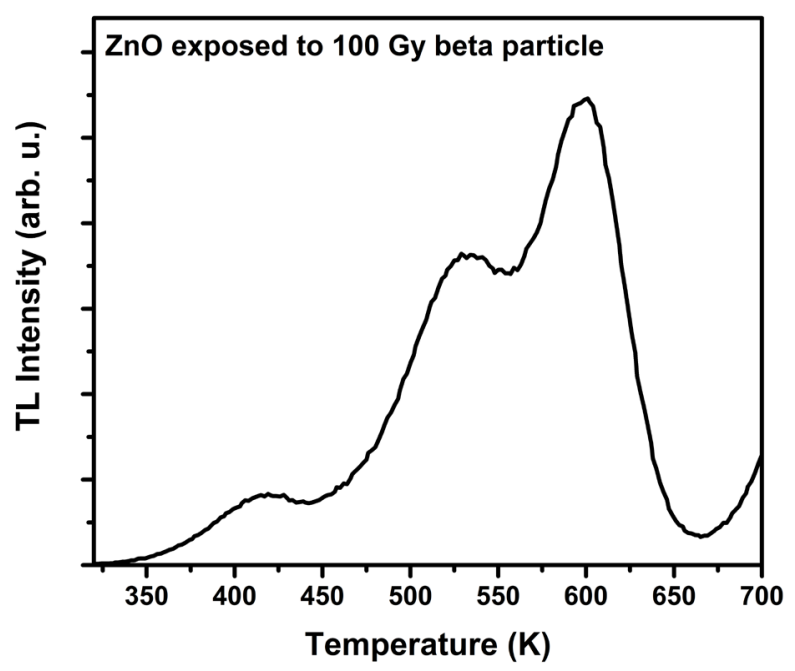

Figure S1. Glow curves of $\mathrm{ZnO}$ exposed to $100 \mathrm{~Gy}$ of beta particle irradiation. 